\title{
Isolated port-site metastasis after surgical staging for low-risk endometrioid endometrial cancer: A case report
}

\author{
DANIELE MAUTONE ${ }^{1}$, ANDREA DALL'ASTA $^{1}$, MICHELA MONICA $^{1}$, LETIZIA GALLI $^{1}$, \\ VITO ANDREA CAPOZZI ${ }^{1}$, FEDERICO MARCHESI ${ }^{2}$, GIOVANNA GIORDANO $^{3}$ and ROBERTO BERRETTA ${ }^{1}$ \\ ${ }^{1}$ Department of Surgical Sciences, Obstetrics and Gynecology Unit; ${ }^{2}$ Department of Surgical Sciences, \\ Gastrointestinal Surgery Unit; ${ }^{3}$ Department of Biomedical, Biotechnological and Translational Sciences, \\ Pathological Anatomy and Histology Unit, University of Parma, Parma I-43126, Italy
}

Received March 21, 2015; Accepted March 9, 2016

DOI: $10.3892 / 01.2016 .4595$

\begin{abstract}
Port-site metastases (PSMs) are well-known potential complications of laparoscopic surgery for gynaecologic malignancies. The present case study reports PSM following laparoscopic surgery for Stage IA Grade 1 endometrioid endometrial cancer (EEC). The recurrence developed within 7 months following primary surgery and required surgical excision followed by adjuvant chemo-radio therapy. After 9 months, the patient remains disease-free. PSMs are rare complications following laparoscopic surgery. Amongst the 23 cases of endometrial cancer PSMs reported so far, only 4 followed EEC Stage IA Grade 1-2. The present study reports a rare case of PSM after Stage IA Grade 1 EEC. The clinical and prognostic relevance of PSMs has not been identified so far; and it is not known whether PSMs represent a local recurrence or a systemic recurrence. Surgeons should be aware that even low-risk EEC may be followed by PSMs and should take steps to prevent these rare recurrences.
\end{abstract}

\section{Introduction}

Endometrial cancer (EC) is the most common malignant tumor of the female genital tract in the developed countries of North America and Europe. At diagnosis, endometrioid endometrial cancer (EEC) does not extend beyond the uterus in $>75 \%$ of cases and is characterized by a good prognosis,

Correspondence to: Dr Andrea Dall'Asta, Department of Surgical Sciences, Obstetrics and Gynecology Unit, University of Parma, 14 Via Antonio Gramsci, Parma I-43126, Italy

E-mail: andrea.dallasta1@gmail.com

Abbreviations: EC, endometrial cancer; EEC, endometrioid endometrial cancer; PSM, port site metastasis; CT, computed tomography; TLH, total laparoscopic hysterectomy; PET, positron emission tomography; BSO, bilateral salpingo-oophorectomy

Key words: gynecology oncology, endometrial cancer, port-site metastasis, laparoscopy with an overall 5-year survival rate of $75-80 \%(1,2)$. Histological diagnosis reports in most cases a type $1 \mathrm{EC}$, termed EEC, which is usually associated with low aggressiveness and long term disease free survival; whereas type 2 EC refers to high-risk neoplasias, such as serous or clear cell EC (1).

The current management of EC includes total hysterectomy with bilateral salpingo-oophorectomy (BSO), lymphadenectomy, if necessary, and peritoneal cytology (3). Laparoscopy offers unique benefits including shorter recovery time, improved performance status following surgery and a decreased risk of adhesions when compared with open laparotomy procedures, and is increasingly used as an alternative to abdominal surgery in the management of EC (4).

The laparoscopic management of malignancies has also brought about novel, late complications of surgical therapy in the form of tumor recurrences and trocar insertion sites metastases (5). Laparoscopic port-site metastases (PSMs) are early recurrent tumorous lesions developing locally in the abdominal wall within the scar tissue of one or more trocar sites, and can be compared to wound metastases after open surgery. By definition, port site recurrences are not associated with diffuse peritoneal carcinomatosis (5-7).

The overall incidence of PSM in gynecologic malignancies has been estimated to be $1-2 \%$, reaching $19.6 \%$ in patients with advanced ovarian cancer undergoing diagnostic laparoscopy; PSMs have also been reported in patients with previous EC (8). The purpose of the present case study is to describe a rare case of isolated port-site metastasis following laparoscopic surgical staging for early low-grade EEC and to review all the published cases of PSMs after laparoscopic surgery for EC.

\section{Case report}

A 57-year-old woman who had been complaining of postmenopausal uterine bleeding for 8 months underwent hysteroscopy without anaesthesia with endometrial biopsy in January 2013 at the University Hospital of Parma (Parma, Italy). The pathology report revealed a grade 1 EEC. The patient's medical history revealed arterial hypertension, diabetes, severe obesity, gastric banding and had 3 previous successful pregnancies and 2 miscarriages. Preoperatory CA125, CA19.9, CA 15.3 and 


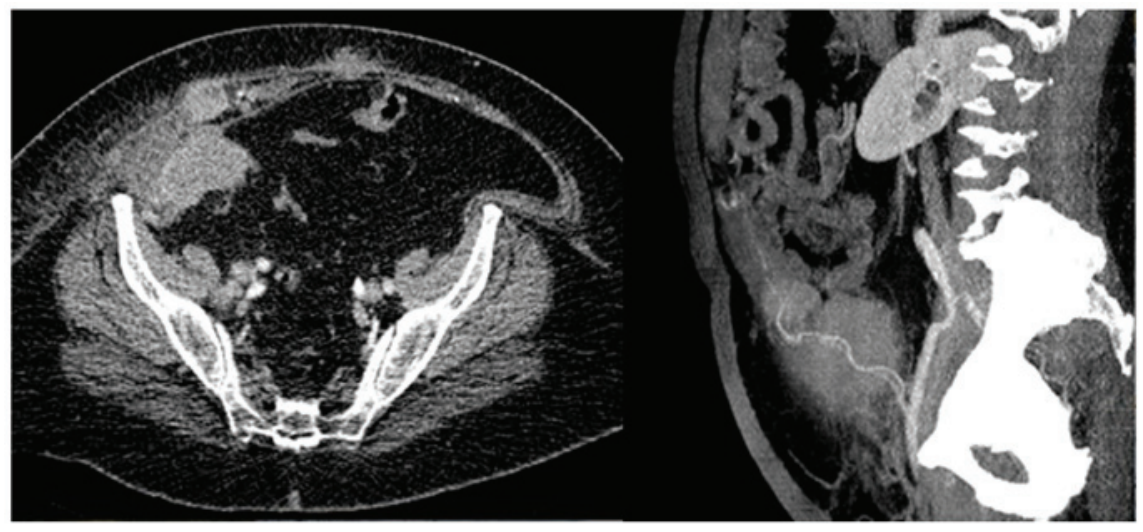

Figure 1. Computed tomography axial (left) and coronal (right) view: The images show multiple nodules with increased vascularization in the context of the right rectus abdominis muscle and a bulky right common iliac lymph node consistent with metastatic lesions.

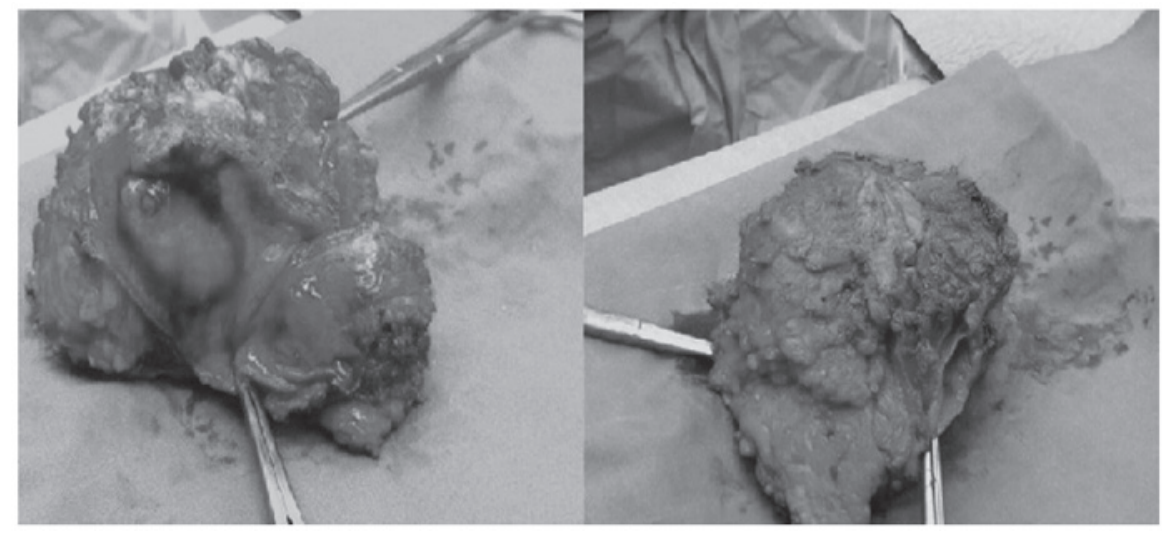

Figure 2. Surgical specimen (largest diameter, $9.5 \times 8 \mathrm{~cm}$ ).

CEA immunopositivity were negative. Preoperative imaging, including transvaginal ultrasound and total body computed tomography (CT) suggested a stage I tumor. In February 2013, a total laparoscopic hysterectomy (TLH) with BSO and pelvic washing were performed.

The surgical procedure started with uterine manipulator placement. A $12 \mathrm{mmHg}$ pneumoperitoneum was induced through a $10-\mathrm{mm}$ optic view umbilical port; three $5-\mathrm{mm}$ additional ports in the right and the left iliac fossa and in the sovrapubic region were placed. The fallopian tubes were coagulated in their uterine proximal portion and peritoneal washing for cytology was performed. A surgical specimen was vaginally extracted without a protection bag. Frozen sections, $10-\mu \mathrm{m}$ thick and stained with hematoxylin and eosin to reveal morphology, revealed an endometrioid grade 1 tumor with myometrial invasion $<50 \%$, thus it was decided that pelvic and paraortic lymph-nodes dissection would not be performed. The ancillary trocars were removed after desufflating through the umbilical port, then the abdominal wall was closed. All port-wounds were irrigated with povidone. No complications occurred during and after the procedure.

The pathology report revealed a FIGO 2009 Stage IA grade 1 EEC with negative pelvic washing and did not indicate a requirement for adjuvant therapy. A total of 7 months after the primary surgery the patient complained of pain in the right iliac fossa near to the port site scar. While examining the patient, a little tender nodule was identified. An abdominal ultrasound and an abdominal CT revealed a 28x24x19 mm irregular solid nodule close to the right rectus abdominis muscle. After 2 months, immunopositivity for CA125 was significantly increased and a CT scan revealed multiple highly vascularized nodules, the largest one being $7 \mathrm{~cm}$ and the second largest was $4 \mathrm{~cm}$, into the right rectus abdominis muscle near to the homologous fascia and a bulky right common iliac lymph node, consistent with metastatic lesions (Fig. 1).

The patient underwent surgery. Peritoneal carcinomatosis was not observed during the laparotomic procedure; the abdominal metastatic lesion with the corresponding fascia and skin was removed (Fig. 2) and the right external lymphnodes were positive on positron emission tomography (PET) scan. The fascia defect was repaired with a synthetic, nonabsorbable polypropylene mesh. The pathology report revealed a metastatic endometrioid Grade 1 tumor; resected edges and the lymph nodes were both negative for neoplastic spread (Fig. 3). The adjuvant treatment comprised chemotherapy with $175 \mathrm{mg} / \mathrm{m}^{2}$ carboplatin AUC 5 and taxolo, which had been stopped after the first cycle because of intolerance (the patient completed 6 cycles monochemotherapy with carboplatin, AUC 5, every 21 days), followed by abdominal external radiotherapy, which remains ongoing. After 9 months, the patient remains disease free and is negative for CA125. 
A

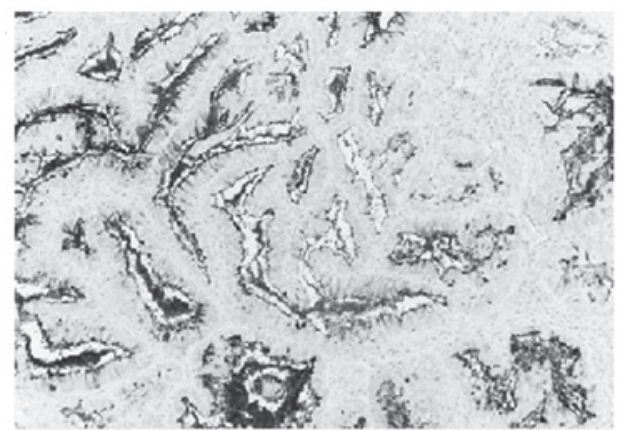

B

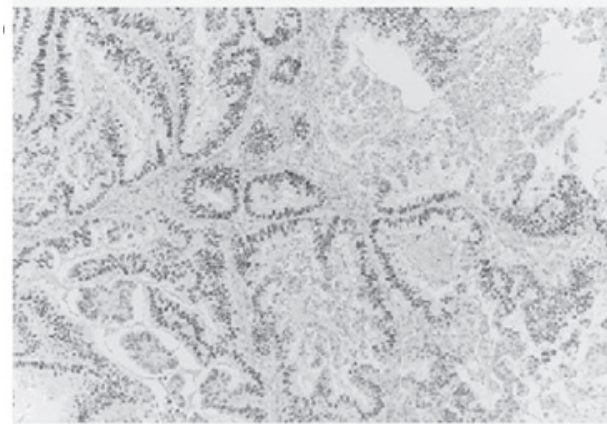

C

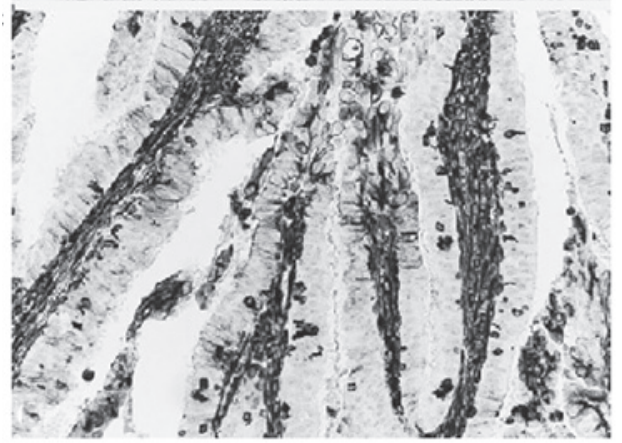

Figure 3. The endometroid nature of the metastatic lesion was demon strated by positivity to (A) CA125, x100; (B) Estrogen receptor, x100 and (C) Vimentin, $\mathrm{x} 200$.

\section{Discussion}

PSMs have been described as a rare phenomena, occurring in $<80$ cases of gynecologic malignancies. Historically the first case of PSM after laparoscopic surgery for EC was reported in 1997 by Kadar et al (9).

A recent review by Palomba et al (8) of published and unpublished work up to 2011 reported only 9 cases of PSMs following laparoscopic surgery for EEC: Amongst those, there was 1 Stage IA Grade 2 case and 3 Stage IB Grade 2 cases according to the FIGO 2009 Classification.

After 2011, Grabosh and Xynos (10) described 2 cases of isolated PSM after robotic surgery for EEC. The first case was a Stage IA Grade 1 EEC treated with TLH, BSO and pelvic lymphadenectomy. The second case was a Stage IA Grade 1-2 EEC treated with TLH, BSO and pelvic and paraortic lymphadenectomy due to uterine perforation during the surgical procedure. Both cases were treated with surgical excision of the PSM followed by chemo- and radiotherapy. Lonnerfors et al (11) reported 4 cases of PSMs, the first one was following a EEC Stage IIIC, the second and the third case were after clear-cell adenocarcinoma Stage III and the fourth case occurred following a carcinosarcoma Stage IB. EEC was treated with laparoscopic radical hysterectomy, BSO, pelvic nodal sampling and adjuvant chemo-, radio- and progesterone therapy. The PSM was diagnosed 19 months after the primary surgery and was associated with nodal and vaginal-cuff metastases. The patient did not undergo additional treatment. Nguyen et al (12) reported a case of PSM after surgical treatment for Stage IB Grade 3 EEC treated with TLH, BSO, pelvic and paraortic lymphadenectomy and brachitherapy. Two PSMs of $1.5 \mathrm{~cm}$ were diagnosed 12 months after surgery in two different port sites and were associated with vaginal cuff metastases. The treatment comprised surgical excision followed by chemotherapy. Rindos et al (13) reported two cases of PSMs after primary surgery for EC. The first case was an isolated PSM 25 months after TLH and BSO for Stage IA Grade 2 EEC treated with surgical excision followed by chemo and radiotherapy; the second case was a Stage 2 Grade 3 EC with sarcomatous component treated with radical hysterectomy and BSO. Finally, Rauff et al (14) reported a case of serous Stage IIIB Grade 3 EC treated with robotic surgery comprising TLH, BSO, pelvic lymphadenectomy and omentectomy, followed by pelvic and vaginal cuff radiotherapy (the patient refused chemotherapy). PSM was diagnosed 5 months after primary surgery and treated with surgical excision and chemotherapy with the addition of radiotherapy due to peritoneal progression of disease during chemotherapy.

The etiology of PSM remains unknown. Factors involved in PSMs may include exfoliation and spread of tumor cells by laparoscopic instruments, direct implantation at the trocar site by frequent changes of instruments, direct implantation from the passage of the specimen, and the pneumoperitoneum itself can create a 'chimney effect' that causes the passage of tumor cells at port-sites. Moreover, laparoscopic port sites and peritoneal incisions have demonstrated rapid cellular turnover and might provide fertile ground for tumor cells. Other risk factors for the development of port site metastases include ascites and advanced stage (9).

According to the recommendations by Ramirez et al (15), PSMs prevention could be achieved through a reduction in tissue trauma and in the number of the transferred instrument, a 5\% povidone-iodine trocar rinsing before insertion and trocar fixation. In addition, it may be useful to rinse the tip of the instruments with $5 \%$ povidone-iodine when interchanging them, to resect the tumor with adequate margins, to use protective retrieval bags, to remove all the intraabdominal fluid before trocar removal, to deflate the abdomen without trocars removal, to irrigate the trocar sites with $5 \%$ povidone-iodine and to close the peritoneal trocar sites (10-12 mm trocars).

PSMs are rare complications following laparoscopic surgery. Stage IA Grade 1 EEC usually has a high survival rate and a low 5-year recurrence risk. Amongst the 23 cases of EC PSMs reported so far, 14 followed EEC and only 4 followed EEC Stage IA Grade 1-2. In the present study, a rare case of PSM after Stage IA Grade 1 EEC was reported. Surgeons must be conscious that PSMs may occur even after low-risk disease and should take steps to prevent them. The clinical and prognostic relevance of PSMs has not been clarified at present as PSMs etiology is unknown; and it is not known whether PSMs represent a local or systemic recurrence. The gold-standard treatment has not been established and in the majority of cases results in a combined approach with surgery, chemo- and radiotherapy. 


\section{References}

1. Prat J: Prognostic parameters of endometrial carcinoma. Hum Pathol 35: 649-662, 2004.

2. Giordano G, D'Adda T, Bottarelli L, Lombardi M, Brigati F, Berretta R and Merisio C: Two cases of low-grade endometriod carcinoma associated with undifferentiated carcinoma of the uterus (dedifferentiated carcinoma): A molecular study. Pathol Oncol Res 18: 523-528, 2012.

3. Sanjuán A, Hernández S, Pahisa J, Ayuso JR, Torné A, Martínez Román S, Lejárcegui JA, Ordi J and Vanrell JA: Port-site metastasis after laparoscopic surgery for endometrial carcinoma: Two case reports. Gynecol Oncol 96: 539-542, 2005.

4. Dargent DF and Plante M: Laparoscopic surgery in gynecologic cancer. In: Principles and practice of gynecologic oncology. Hoskins WJ, Perez CA and Young RC (eds). 3rd edition. Lippincott Williams \& Wilkins Philadelphia, PA, pp265-295, 2000.

5. Schneider C, Jung A, Reymond MA, Tannapfel A, Balli J, Franklin ME, Hohenberger W and Köckerling F: Efficacy of surgical measures in preventing port site recurrences in a porcine model. Surg Endosc 15: 121-125, 2001.

6. Castillo OA and Vitagliano G: Port site metastasis and tumor seeding in oncologic laparoscopic urology. Urology 71: 372-378, 2008.

7. Lee BR, Tan BJ and Smith AD: Laparoscopic port site metastases: Incidence, risk factors, and potential preventive measures. Urology 65: 639-644, 2005.
8. Palomba S, Falbo A and Zullo F: Isolated port-site metastasis after laparoscopic surgery for endometrial cancer: A case report. Gynecol Oncol Case Rep 2: 16-17, 2012.

9. Kadar N: Port-site recurrences following laparoscopic operations for gynaecological malignancies. Br J Obstet Gynaecol 104: 1308-1313, 1997.

10. Grabosch S and Xynos F: Isolated port-site metastasis after robotic hysterectomy for stage IA endometrial adenocarcinoma. Obstet Gynecol 122: 437-439, 2013.

11. Lönnerfors C, Bossmar T and Persson J: Port-site metastases following robot-assisted laparoscopic surgery for gynecological malignancies. Acta Obstet Gynecol Scand 92: 1361-1368, 2013.

12. Nguyen T, Friedman J, Pradhan TS, Pua TL and Tedjarati SS: Abdominal wall port site metastasis after robotically staged endometrial carcinoma: A case report. Int J Surg Case Rep 4: 613-615, 2013.

13. Rindos N, Curry CL, Tabbarah R and Wright V: Port-site metastases after robotic surgery for gynecologic malignancy. JSLS 18: 66-70, 2014.

14. Rauff S and Ng JS: Port-site recurrence in a patient undergoing robot-assisted gynecologic cancer surgery for endometrial cancer-A case report. Gynecol Oncol Case Rep 2: 127-129, 2012.

15. Ramirez PT, Frumovitz M, Wolf JK and Levenback C: Laparoscopic port-site metastases in patients with gynecological malignancies. Int J Gynecol Cancer 14: 1070-1077, 2004. 